Research Article

\title{
Divisor Problems Related to Hecke Eigenvalues in Three Dimensions
}

\author{
Jing Huang and Huafeng Liu \\ School of Mathematics and Statistics, Shandong Normal University, Jinan, Shandong 250014, China \\ Correspondence should be addressed to Huafeng Liu; hfliu_sdu@hotmail.com
}

Received 26 March 2021; Revised 4 April 2021; Accepted 7 April 2021; Published 20 April 2021

Academic Editor: Jie $\mathrm{Wu}$

Copyright (c) 2021 Jing Huang and Huafeng Liu. This is an open access article distributed under the Creative Commons Attribution License, which permits unrestricted use, distribution, and reproduction in any medium, provided the original work is properly cited.

In this paper, we consider divisor problems related to Hecke eigenvalues in three dimensions. We establish upper bounds and asymptotic formulas for these problems on average.

\section{Introduction}

Let $\Gamma=S L_{2}(\mathbb{Z})$ be the full modular group. Let $H_{k}^{*}$ denote the set of primitive holomorphic forms with even integral weight $k \geq 2$ for $\Gamma$. Then $H_{k}^{*}$ consists of common eigenfunctions $f$ of all Hecke operators $T_{n}$. The Hecke eigenfunction $f$ has the following Fourier series expansion:

$$
f(z)=\sum_{n=1}^{\infty} \lambda_{f}(n) n^{(k-1) / 2} e^{2 \pi i n z}, \quad(\Im z>0),
$$

where $\lambda_{f}(n)$ denotes the $n$-th normalized eigenvalue. It is known that $\lambda_{f}(n)$, as a function of $n$, is real-valued and multiplicative. Moreover, for all integers $n \geq 1$, Deligne [1] showed that

$$
\left|\lambda_{f}(n)\right| \leq d(n),
$$

where $d(n)$ is the divisor function. Also, for every prime $p$,

$$
\begin{gathered}
\alpha_{f}(p)=\alpha_{f}(p)+\beta_{f}(p), \\
\alpha_{f}(p) \beta_{f}(p)=\left|\alpha_{f}(p)\right|=\left|\beta_{f}(p)\right|=1 .
\end{gathered}
$$

Now we introduce some specific automorphic $L$-functions. Define the Hecke $L$-function attached to $f$ as

$$
L(s, f)=\sum_{n=1}^{\infty} \frac{\lambda_{f}(n)}{n^{s}}=\prod_{p}\left(1-\frac{\alpha_{f}(p)}{p^{s}}\right)^{-1}\left(1-\frac{\alpha_{f}(p)^{-1}}{p^{s}}\right)^{-1},
$$

for $\Re s>1$. Moreover, the Rankin-Selberg $L$-function attached to $f$ can be defined as

$$
\begin{aligned}
L(s, f \times f)= & \prod_{p}\left(1-\frac{\alpha_{f}(p)^{2}}{p^{s}}\right)^{-1}\left(1-\frac{1}{p^{s}}\right)^{-2} \\
& \cdot\left(1-\frac{\alpha_{f}(p)^{-2}}{p^{s}}\right)^{-1}
\end{aligned}
$$

$$
\Re s>1 .
$$

Then, $L(s, f \times f)$ can be rewritten as

$$
L(s, f \times f)=\zeta(2 s) \sum_{n=1}^{\infty} \frac{\lambda_{f}(n)^{2}}{n^{s}}:=\sum_{n=1}^{\infty} \frac{\lambda_{f \times f}(n)}{n^{s}} .
$$

As usual, $\zeta(s)$ denotes the Riemann zeta-function. The symmetric square $L$-function attached to $f$ can be defined as, for $\mathfrak{R} s>1$, 


$$
L\left(s, \operatorname{sym}^{2} f\right)=\prod_{p}\left(1-\frac{\alpha_{f}(p)^{2}}{p^{s}}\right)^{-1}\left(1-\frac{1}{p^{s}}\right)^{-1}\left(1-\frac{\alpha_{f}(p)^{-2}}{p^{s}}\right)^{-1},
$$

which can also be expressed in the Dirichlet series

$$
\begin{aligned}
L\left(s, \operatorname{sym}^{2} f\right) & =\sum_{n=1}^{\infty} \frac{\lambda_{\mathrm{sym}^{2} f}(n)}{n^{s}} \\
& =\prod_{p}\left(1+\frac{\lambda_{\text {sym }^{2} f}(p)}{p^{s}}+\frac{\lambda_{\text {sym }^{2} f}\left(p^{2}\right)}{p^{2 s}}+\frac{\lambda_{\text {sym }^{2} f}\left(p^{3}\right)}{p^{3 s}}+\cdots\right), \quad \Re s>1 .
\end{aligned}
$$

The symmetric square $L$-function $L\left(s, \operatorname{sym}^{2} f\right)$ has a functional equation and could be analytic continued to an entire function over the whole complex plane. We refer to works of Hecke in [2], Gelbert and Jacquet [3], Kim [4], and Kim and Shahidi $[5,6]$ for these properties. Therefore, the symmetric square $L$-function $L\left(s, \operatorname{sym}^{2} f\right)$ could be seen as a general $L$-function in the sense of Perelli [7].

In number theory, considering the properties and average behaviors of the Fourier coefficients is meaningful and interesting. Some classical problems concern mean values of these Fourier coefficients and related problems with the corresponding automorphic $L$-functions (see [1, 8-29], etc.). Here, we just give a brief history for general divisor problems related to these Fourier coefficients.

Let $\omega$ be an integer greater than one, and

$$
\begin{aligned}
\lambda_{\omega, f}(n) & =\sum_{n=n_{1} n_{2}, \ldots, n_{\omega}} \lambda_{f}\left(n_{1}\right) \lambda_{f}\left(n_{2}\right), \ldots, \lambda_{f}\left(n_{\omega}\right), \\
\lambda_{\omega, f \times f}(n) & =\sum_{n=n_{1} n_{2}, \ldots, n_{\omega}} \lambda_{f \times f}\left(n_{1}\right) \lambda_{f \times f}\left(n_{2}\right), \ldots, \lambda_{f \times f}\left(n_{\omega}\right) .
\end{aligned}
$$

In particular, we have $\lambda_{1, f}(n)=\lambda_{f}(n)$ and $\lambda_{1, f \times f}(n)=$ $\lambda_{f \times f}(n)$. In 1927, Hecke [30] showed that

$$
\sum_{n \leq x} \lambda_{f}(n) \ll x^{(1 / 2)}
$$

Subsequently, this upper bound was improved by many scholars (for example, see $[12,21,24]$ ). In this direction, the best result so far was obtained by $\mathrm{Wu}$ [24] who showed that

$$
\sum_{n \leq x} \lambda_{f}(n) \ll x^{(1 / 3)}(\log x)^{\rho_{(1 / 2)}^{+}}
$$

where

$$
\begin{aligned}
\rho_{(1 / 2)}^{+}= & \frac{102+7 \sqrt{21}}{210}\left(\frac{6-\sqrt{21}}{5}\right)^{(1 / 2)} \\
& +\frac{102-7 \sqrt{21}}{210}\left(\frac{6+\sqrt{21}}{5}\right)^{(1 / 2)}-\frac{33}{55}, \\
= & -0.118 \ldots
\end{aligned}
$$

Rankin [20] and Selberg [22] established

$$
\sum_{n \leq x} \lambda_{f \times f}(n)=C_{f} x+O_{f}\left(x^{(3 / 5)}\right),
$$

where $C_{f}$ is a positive constant depending on $f$. Later, Kanemitsu, Sankaranarayanan, and Tanigawa [31] studied general divisor problems and proved

$$
\begin{aligned}
\sum_{n \leq x} \lambda_{\omega, f}(n) & \ll x^{1-(3 / 2 k+2)+\varepsilon}, \\
\sum_{n \leq x} \lambda_{\omega, f \times f}(n) & =M_{\omega}(x)+O\left(x^{1-(1 / 2 k)+\varepsilon}\right),
\end{aligned}
$$

where $\omega$ is an integer and $\omega \geq 2 ; M_{\omega}(x)$ comes from a residue and takes the form $x P_{\omega-1}(\log x) ; P_{\omega-1}(t)$ denotes a polynomial of $t$ with degree $\omega-1$. Fomenko [32] also proved the same result for the sum $\sum_{n \leq x} \lambda_{\omega, f}(n)$. After that, Kanemitsu, Sankaranarayanan, and Tanigawa's result was improved by Lü [33], and some general results were obtained (see [34-37], etc.).

In this paper, we consider divisor problems related to Hecke eigenvalues in three dimensions motivated by the above results and Ivić's work on three-dimensional divisor problems (see, e.g., [38]). We first introduce some notation. For any fixed integer $1<a<b<c$, we define

$$
\begin{aligned}
& \lambda_{f}^{a, b, c}(n):=\sum_{n=n_{1}^{a} n_{2}^{b} n_{3}^{c}} \lambda_{f}\left(n_{1}\right) \lambda_{f}\left(n_{2}\right) \lambda_{f}\left(n_{3}\right), \\
& \lambda_{f \times f}^{a, b, c}(n):=\sum_{n=n_{1}^{a} n_{2}^{b} n_{3}^{c}} \lambda_{f \times f}\left(n_{1}\right) \lambda_{f \times f}\left(n_{2}\right) \lambda_{f \times f}\left(n_{3}\right) .
\end{aligned}
$$

We are interested in studying the average behaviors of sums

$$
\begin{aligned}
S_{f}(a, b, c ; x) & :=\sum_{n \leq x} \lambda_{f}^{a, b, c}(n), \\
S_{f \times f}(a, b, c ; x) & :=\sum_{n \leq x} \lambda_{f \times f}^{a, b, c}(n),
\end{aligned}
$$

which can be seen as divisor problems related to Hecke eigenvalues in three dimensions. We establish the following results. 
Theorem 1. Let $a, b$, and $c$ be fixed integers satisfying $1<a<b<c$. Then, for any $\epsilon>0$, one has

$$
S_{f}(a, b, c ; x) \ll \begin{cases}x^{(1 / a)-(3 / 2(7 a-b-c))+\varepsilon}, & \text { if } c \leq 2 a, \\ x^{(1 / a)-(3 / 2(5 a-b))+\varepsilon}, & \text { if } b<2 a<c, \\ x^{(1 / 2 a)+\varepsilon}, & \text { if } 2 a \leq b .\end{cases}
$$

Theorem 2. Let $a, b$, and $c$ be fixed integers satisfying $1<a<b<c$. Then, for any $\epsilon>0$, one has

$$
S_{f \times f}(a, b, c ; x)= \begin{cases}M_{1} x^{(1 / a)}+M_{2} x^{(1 / b)}+M_{3} x^{(1 / c)}+O\left(x^{(1 / a)-(84 / 748 a-131(b+c))+\varepsilon}\right), & \text { if } c \leq 2 a, \\ M_{1} x^{(1 / a)}+M_{2} x^{(1 / b)}+O\left(x^{(1 / a)-(84 / 486 a-131 b)+\varepsilon}\right), & \text { if } b<2 a<c, \\ M_{1} x^{(1 / a)}+O\left(x^{(5 / 8 a)+\varepsilon}\right), & \text { if } 2 a \leq b,\end{cases}
$$

where

$$
\begin{aligned}
& M_{1}=L\left(\frac{b}{a}, f \times f\right) L\left(\frac{c}{a}, f \times f\right) L\left(1, \operatorname{sym}^{2} f\right), \\
& M_{2}=L\left(\frac{a}{b}, f \times f\right) L\left(\frac{c}{b}, f \times f\right) L\left(1, \operatorname{sym}^{2} f\right), \\
& M_{3}=L\left(\frac{a}{c}, f \times f\right) L\left(\frac{b}{c}, f \times f\right) L\left(1, \operatorname{sym}^{2} f\right) .
\end{aligned}
$$

\section{Some Lemmas}

To prove our theorems, we need to quote some lemmas, which include the individual and averaged subconvexity bounds for the Riemann zeta-function and the symmetric square $L$-function. And from the following Lemma 1, we know that the Rankin-Selberg $L$-function $L(s, f \times f)$ could be decomposed into the product of the Riemann zetafunction and the corresponding symmetric square $L$-function.

Lemma 1. For $\mathfrak{R} s>1$, one has

$$
L(s, f \times f)=\zeta(s) L\left(s, \operatorname{sym}^{2} f\right) .
$$

Proof. By comparing the Euler products of two sides of (20) and applying (3), we can get this lemma. This lemma can also be found in [33].

Lemma 2. For any $\epsilon>0$, one has

$$
\int_{1}^{T}\left|L\left(\frac{1}{2}+i t, f\right)\right|^{2} \mathrm{~d} t \sim C T \log T
$$

uniformly for $T \geq 1$, and the subconvexity bound in the critical strip

$$
L(\sigma+i t, f) \ll \begin{cases}(1+|t|)^{(2(1-\sigma) / 3)+\varepsilon}, & \text { if } \frac{1}{2}<\sigma \leq 1, \\ 1, & \text { if } \sigma>1,\end{cases}
$$

where $|t| \geq 1$.

Proof. These results are proved by Good [11].

Lemma 3. For any $\varepsilon>0$, one has

$$
\int_{1}^{T}\left|\zeta\left(\frac{1}{2}+i t\right)\right|^{12} \mathrm{~d} t \ll T^{2+\varepsilon}
$$

uniformly for $T \geq 1$, and the subconvexity bound in the critical strip

$$
\zeta(\sigma+i t) \ll \begin{cases}(1+|t|)^{(13 / 42)(1-\sigma)+\varepsilon}, & \text { if } \frac{1}{2} \leq \sigma \leq 1, \\ 1, & \text { if } \sigma>1,\end{cases}
$$

where $|t| \geq 1$.

Proof. The twelfth mean value estimate (23) is due to HeathBrown [39]. The subconvexity bound (24) is due to Bourgain [40].

Lemma 4. For any $\epsilon>0$, one has

$$
\int_{1}^{T}\left|L\left(\sigma+i t, \operatorname{sym}^{2} f\right)\right|^{2} \mathrm{~d} t \ll T^{3(1-\sigma)+\varepsilon}
$$

uniformly for $T \geq 1$, and the subconvexity bound in the critical strip

$$
L\left(\sigma+i t, \operatorname{sym}^{2} f\right) \ll \begin{cases}(1+|t|)^{(5 / 4)(1-\sigma)+\varepsilon}, & \text { if } \frac{1}{2} \leq \sigma \leq 1, \\ 1, & \text { if } \sigma>1,\end{cases}
$$

where $|t| \geq 1$. 
Proof. The first result follows from Perelli's mean value theorem with $L\left(s, \operatorname{sym}^{2} f\right)$ (see [7]), and the second one is due to Nunes [19].

\section{Proof of Theorem 1}

In this section, we shall complete the proof of Theorem 1. Note that

$$
L(a s, f) L(b s, f) L(c s, f)=\sum_{n=1}^{\infty} \frac{\lambda_{f}^{a, b, c}(n)}{n^{s}} .
$$

Then, by (27) and Perron's formula (see Proposition 5.54 in [41]), we can obtain

$$
\begin{aligned}
S_{f}(a, b, c ; x)= & \frac{1}{2 \pi i} \int_{\eta-i T}^{\eta+i T} L(a s, f) L(b s, f) L(c s, f) \\
& \frac{x^{s}}{s} \mathrm{~d} s+O\left(\frac{x^{(1 / a)+\varepsilon}}{T}\right),
\end{aligned}
$$

where $s=\sigma+i t, \eta=(1 / a)+\varepsilon$ and $1 \leq T \leq x$ is a parameter to be chosen later.

We shift the line of the integral of (28) to the line $\Re s=(1 / 2 a)$. Then, Cauchy's residue theorem shows that

$$
\begin{aligned}
S_{f}(a, b, c ; x)= & \frac{1}{2 \pi i}\left\{\int_{(1 / 2 a)-i T}^{(1 / 2 a)+i T}+\int_{(1 / 2 a)+i T}^{\eta+i T}+\int_{\eta-i T}^{(1 / 2 a)-i T}\right\} \\
& L(a s, f) L(b s, f) L(c s, f) \frac{x^{s}}{s} d s+O\left(\frac{x^{(1 / a)+\varepsilon}}{T}\right) \\
:= & G_{1}+G_{2}+G_{3}+O\left(\frac{x^{(1 / a)+\varepsilon}}{T}\right) .
\end{aligned}
$$

The following work is to estimate $G_{1}, G_{2}$, and $G_{3}$. The estimates for the integrals over the horizontal segments are similar, so we handle $G_{2}$ and $G_{3}$ first. To get this goal, we consider three cases $c \leq 2 a, b<2 a<c$, and $2 a \leq b$.

We first consider the case $c \leq 2 a$. To estimate $G_{2}$ and $G_{3}$, we divide the integral interval into the following eight intervals $I_{1}, \ldots, I_{4}$, some of which may be empty, and use Lemma 2.

Interval $I_{1}$ :

$$
\begin{aligned}
I_{1} & :=\left\{s=\sigma+i T: \frac{1}{2} \leq a \sigma \leq 1, \frac{b}{2 a} \leq b \sigma \leq 1, \frac{c}{2 a} \leq c \sigma \leq 1\right\} \\
& =\left\{s=\sigma+i T: \frac{1}{2 a} \leq \sigma \leq \frac{1}{c}\right\} .
\end{aligned}
$$

In this interval, we have

$$
\begin{aligned}
T^{-1} & \times \int_{I_{1}} x^{\sigma}|L(a \sigma+i a t, f) L(b \sigma+i b t, f) L(c \sigma+i c t, f)| \mathrm{d} \sigma \\
& \ll \max _{(1 / 2 a) \leq \sigma \leq(1 / c)} x^{\sigma} T^{(2 / 3)(1-a \sigma)+(2 / 3)(1-b \sigma)+(2 / 3)(1-c \sigma)+\varepsilon} T^{-1} \\
& \ll \max _{(1 / 2 a) \leq \sigma \leq(1 / c)} T^{1+\varepsilon}\left(\frac{x}{T^{(2 / 3)(a+b+c)}}\right)^{\sigma} \\
& \ll x^{(1 / c)} T^{(1 / 3)-(2(a+b) / 3 c)+\varepsilon}+x^{(1 / 2 a)} T^{(2 / 3)-(b+c / 3 a)+\varepsilon} .
\end{aligned}
$$

Interval $I_{2}$ :

$$
\begin{aligned}
I_{2} & :=\left\{s=\sigma+i T: \frac{1}{2} \leq a \sigma \leq 1, \frac{b}{2 a} \leq b \sigma \leq 1,1<c \sigma \leq c \eta\right\} \\
& =\left\{s=\sigma+i T: \frac{1}{c}<\sigma \leq \frac{1}{b}\right\} .
\end{aligned}
$$

In this interval, we have

$$
\begin{aligned}
T^{-1} & \times \int_{I_{2}} x^{\sigma}|L(a \sigma+i a t, f) L(b \sigma+i b t, f) L(c \sigma+i c t, f)| \mathrm{d} \sigma \\
& \ll \max _{(1 / c)<\sigma \leq(1 / b)} x^{\sigma} T^{(2 / 3)(1-a \sigma)+(2 / 3)(1-b \sigma)+\varepsilon} T^{-1} \\
& \ll \max _{(1 / c)<\sigma \leq(1 / b)} T^{(1 / 3)+\varepsilon}\left(\frac{x}{T^{(2 / 3)(a+b)}}\right)^{\sigma} \\
& \ll x^{1 / b} T^{-(1 / 3)-(2 a / 3 b)+\varepsilon}+x^{(1 / c)} T^{(1 / 3)-(2(a+b) / 3 c)+\varepsilon} .
\end{aligned}
$$

Interval $I_{3}$ :

$$
\begin{aligned}
I_{3} & :=\left\{s=\sigma+i T: \frac{1}{2} \leq a \sigma \leq 1,1<b \sigma \leq b \eta, \frac{c}{2 a} \leq c \sigma \leq 1\right\} \\
& =\left\{s=\sigma+i T: \frac{1}{b}<\sigma \leq \frac{1}{c}\right\} .
\end{aligned}
$$

This interval is an empty set noting that $(1 / b)>(1 / c)$. Interval $I_{4}$ :

$$
\begin{aligned}
I_{4} & :=\left\{s=\sigma+i T: \frac{1}{2} \leq a \sigma \leq 1,1<b \sigma \leq b \eta, 1<c \sigma \leq c \eta\right\} \\
& =\left\{s=\sigma+i T: \frac{1}{b}<\sigma \leq \frac{1}{a}\right\} .
\end{aligned}
$$

In this interval, we have 


$$
\begin{aligned}
T^{-1} & \times \int_{I_{4}} x^{\sigma}|L(a \sigma+i a t, f) L(b \sigma+i b t, f) L(c \sigma+i c t, f)| \mathrm{d} \sigma \\
& \ll \max _{(1 / b)<\sigma \leq(1 / a)} x^{\sigma} T^{(2 / 3)(1-a \sigma)+\varepsilon} T^{-1} \\
& \ll x^{(1 / a)} T^{-1+\varepsilon}+x^{(1 / b)} T^{-(1 / 3)-} \frac{2 a}{3 b}+\varepsilon
\end{aligned}
$$

$$
\begin{aligned}
\left|G_{2}+G_{3}\right| & \ll T^{-1} \int_{(1 / 2 a)}^{\eta} x^{\sigma}|L(a \sigma+i a t, f) L(b \sigma+i b t, f) L(c \sigma+i c t, f)| \mathrm{d} \sigma \\
& =T^{-1} \int_{I_{1} \cup \cdots \cup I_{4}} x^{\sigma}|L(a \sigma+i a t, f) L(b \sigma+i b t, f) L(c \sigma+i c t, f)| \mathrm{d} \sigma \\
& \ll x^{(1 / 2 a)} T^{(2 / 3)-(b+c / 3 a)+\varepsilon}+x^{(1 / b)} T^{-(1 / 3)-(2 a / 3 b)+\varepsilon}+x^{(1 / c)} T^{(1 / 3)-(2(a+b) / 3 c)+\varepsilon}+x^{(1 / a)+\varepsilon} T^{-1+\varepsilon} .
\end{aligned}
$$

Next, we handle $G_{1}$. We have

$$
\begin{aligned}
\left|G_{1}\right| & \ll x^{1 / 2 a} \int_{1}^{T}\left|L\left(\frac{1}{2}+i a t, f\right) L\left(\frac{b}{2 a}+i b t, f\right) L\left(\frac{c}{2 a}+i c t, f\right)\right| t^{-1} \mathrm{~d} t+x^{1 / 2 a} \\
& \ll x^{1 / 2 a} \log T \max _{T_{1} \leq T} T_{1}^{-1} \int_{T_{1} / 2}^{T_{1}}\left|L\left(\frac{1}{2}+i a t, f\right) L\left(\frac{b}{2 a}+i b t, f\right) L\left(\frac{c}{2 a}+i c t, f\right)\right| \mathrm{d} t+x^{1 / 2 a} .
\end{aligned}
$$

Then, by Lemma 2 and applying Cauchy's inequality, we can obtain

$$
\begin{aligned}
\left|G_{1}\right| \ll & x^{1 / 2 a} \log T \max _{T_{1} \leq T} T_{1}^{-1} T_{1}^{(2 / 3)(1-(b / 2 a))} T_{1}^{(2 / 3)(1-(c / 2 a))} \\
& \cdot\left(\int_{T_{1} / 2}^{T_{1}}\left|L\left(\frac{1}{2}+i a t, f\right)\right|^{2} \mathrm{~d} t\right)^{1 / 2} \times\left(\int_{T_{1} / 2}^{T_{1}} 1 \mathrm{~d} t\right)^{1 / 2}+x^{1 / 2 a} \\
& \ll x^{1 / 2 a} \log T \max _{T_{1} \leq T} T_{1}^{(4 / 3)-(b+c / 3 a)+\varepsilon} \\
& \ll x^{1 / 2 a} T^{(4 / 3)-(b+c / 3 a)+\varepsilon} .
\end{aligned}
$$

According to (29), (37), and (39), we have

$$
\begin{aligned}
S_{f}(a, b, c ; x) \ll & x^{1 / 2 a} T^{(4 / 3)-(b+c / 3 a)+\varepsilon}+x^{1 / b} T^{-(1 / 3)-(2 a / 3 b)} \\
& +x^{(1 / a)+\varepsilon} T^{-1+\varepsilon} .
\end{aligned}
$$

By taking $T=x^{(3 / 2(7 a-b-c))}$ in (40), we can obtain

$$
S_{f}(a, b, c ; x) \ll x^{(1 / a)-(3 / 2(7 a-b-c))+\varepsilon},
$$

which proves the first result of Theorem 1 .

For the case $b<2 a<c$, to estimate $G_{2}$ and $G_{3}$, we still divide the integral interval into four corresponding short intervals $I_{1}^{\prime \prime}, \ldots, I_{4}^{\prime \prime}$, which are different from ones for the case $c \leq 2 a$. In fact, the corresponding short intervals $I_{1}^{\prime \prime}, I_{3}^{\prime \prime}$ become empty sets in the current situation. However, we still can estimate $G_{2}+G_{3}$ similar to the corresponding parts of the case $c \leq 2 a$ and get

$$
\begin{aligned}
\left|G_{2}+G_{3}\right| \ll & x^{1 / 2 a} T^{-(b / 3 a)+\varepsilon}+x^{1 / b} T^{-(1 / 3)-(2 a / 3 b)+\varepsilon} \\
& +x^{(1 / a)+\varepsilon} T^{-1+\varepsilon} .
\end{aligned}
$$

The estimate of $G_{1}$ becomes the following at the current case by noting $(c / 2 a)>1$. 


$$
\begin{aligned}
\left|G_{1}\right| & \ll x^{1 / 2 a} \int_{1}^{T}\left|L\left(\frac{1}{2}+i a t, f\right) L\left(\frac{b}{2 a}+i b t, f\right) L\left(\frac{c}{2 a}+i c t, f\right)\right| t^{-1} \mathrm{~d} t+x^{1 / 2 a} \\
& \ll x^{1 / 2 a} \log T \lim _{T_{1} \leq T} T_{1}^{-1} \int_{T_{1} / 2}^{T_{1}}\left|L\left(\frac{1}{2}+i a t, f\right) L\left(\frac{b}{2 a}+i b t, f\right) L\left(\frac{c}{2 a}+i c t, f\right)\right| \mathrm{d} t+x^{1 / 2 a} \\
& \ll x^{1 / 2 a} \log T \lim _{T_{1} \leq T} T_{1}^{-1+(2 / 3)(1-(b / 2 a))}\left(\int_{T_{1} / 2}^{T_{1}}\left|L\left(\frac{1}{2}+i a t, f\right)\right|^{2} \mathrm{~d} t\right)^{1 / 2}\left(\int_{T_{1} / 2}^{T_{1}} 1 \mathrm{~d} t\right)^{1 / 2}+x^{1 / 2 a} \\
& \ll x^{1 / 2 a} \log T \lim _{T_{1} \leq T} T_{1}^{-1+(2 / 3)(1-(b / 2 a))+(1 / 2)+(1 / 2)+\varepsilon}+x^{1 / 2 a} \\
& \ll x^{1 / 2 a} T^{(2 / 3)-(b / 3 a)+\varepsilon} .
\end{aligned}
$$

Recalling (29), we have

$$
\begin{aligned}
S_{f}(a, b, c ; x) \ll & x^{1 / 2 a} T^{(2 / 3)-(b / 3 a)+\varepsilon}+x^{1 / b} T^{-(1 / 3)-(2 a / 3 b)+\varepsilon} \\
& +x^{(1 / a)+\varepsilon} T^{-1+\varepsilon} .
\end{aligned}
$$

By taking $T=x^{(3 / 2(5 a-b))}$ in (44), we can get

$$
S_{f}(a, b, c ; x) \ll x^{(1 / a)-(3 / 2(5 a-b))+\varepsilon},
$$

which proves the second result of Theorem 1 .
For the case $2 a \leq b$, to estimate $G_{2}$ and $G_{3}$, we also divide the integral interval into four corresponding short intervals $I_{1}^{\prime}, \ldots, I_{4}^{\prime}$, which are different from ones for the case $c \leq 2 a$. In fact, the corresponding short intervals $I_{1}^{\prime}, I_{2}^{\prime}, I_{3}^{\prime}$ become empty sets in the current situation. However, we still can estimate $G_{2}+G_{3}$ similarly to the corresponding parts of the case $c \leq 2 a$ and get

$$
\left|G_{2}+G_{3}\right| \ll x^{1 / 2 a} T^{-(2 / 3)+\varepsilon}+x^{(1 / a)+\varepsilon} T^{-1+\varepsilon} .
$$

The estimate of $G_{1}$ becomes the following by noting $(c / 2 a)>(b / 2 a)>1$.

$$
\begin{aligned}
\left|G_{1}\right| & \ll x^{1 / 2 a} \int_{1}^{T}\left|L\left(\frac{1}{2}+i a t, f\right) L\left(\frac{b}{2 a}+i b t, f\right) L\left(\frac{c}{2 a}+i c t, f\right)\right| t^{-1} \mathrm{~d} t+x^{1 / 2 a} \\
& \ll x^{1 / 2 a} \log T \max _{T_{1} \leq T} T_{1}^{-1} \int_{T_{1} / 2}^{T_{1}}\left|L\left(\frac{1}{2}+i a t, f\right) L\left(\frac{b}{2 a}+i b t, f\right) L\left(\frac{c}{2 a}+i c t, f\right)\right| \mathrm{d} t+x^{1 / 2 a} \\
& \ll x^{1 / 2 a} \log T \max _{T_{1} \leq T} T_{1}^{-1}\left(\int_{T_{1} / 2}^{T_{1}}\left|L\left(\frac{1}{2}+i a t, f\right)\right|^{2} \mathrm{~d} t\right)^{1 / 2}\left(\int_{\frac{T_{1}}{2}}^{T_{1}} d t\right)^{1 / 2}+x^{1 / 2 a} \\
& \ll x^{1 / 2 a} \log T \max _{T_{1} \leq T} T_{1}^{-1+(1 / 2)+(1 / 2)+\varepsilon}+x^{1 / 2 a} \\
& \ll x^{1 / 2 a} T^{\varepsilon} .
\end{aligned}
$$

Recalling (29), we have

$$
S_{f}(a, b, c ; x) \ll x^{1 / 2 a} T^{\varepsilon}+x^{(1 / a)+\varepsilon} T^{-1+\varepsilon} .
$$

By taking $T=x^{1 / 2 a}$ in (48), we can get

$$
S_{f}(a, b, c ; x) \ll x^{(1 / 2 a)+\varepsilon},
$$

which proves the third result of Theorem 1 .

\section{Proof of Theorem 2}

In this section, we shall prove Theorem 2, the process of which is more complicated than Theorem 1 . Note that

$$
L(a s, f \times f) L(b s, f \times f) L(c s, f \times f)=\sum_{n=1}^{\infty} \frac{\lambda_{f \times f}^{a, b, c}(n)}{n^{s}}
$$

Then, by (50) and Perron's formula (see Proposition 5.54 in [41]), we have

lopenup3

$$
\begin{aligned}
S_{f \times f}(a, b, c ; x)= & \frac{1}{2 \pi i} \int_{\eta-i T}^{\eta+i T} L(a s, f \times f) L(b s, f \times f) L \\
& \cdot(c s, f \times f) \frac{x^{s}}{s} d s+O\left(\frac{x^{(1 / a)+\varepsilon}}{T}\right),
\end{aligned}
$$


where $s=\sigma+i t, \eta=(1 / a)+\varepsilon$ and $1 \leq T \leq x$ is a parameter which will be decided later.

In view of $(20)$, the points $s=(1 / a), s=(1 / b)$ and $s=$ $(1 / c)$ are the only three possible simple poles of the integrand in the rectangle $I_{T}=\{s=\sigma+i t:(1 / 2 a) \leq$ $\sigma \leq \eta,|t| \leq T\}$. The corresponding possible residues at $s=(1 / a), s=(1 / b)$, and $s=(1 / c)$ are equal to

$$
\begin{aligned}
& M_{1}:=L\left(\frac{b}{a}, f \times f\right) L\left(\frac{c}{a}, f \times f\right) L\left(1, \operatorname{sym}^{2} f\right), \\
& M_{2}:=L\left(\frac{a}{b}, f \times f\right) L\left(\frac{c}{b}, f \times f\right) L\left(1, \operatorname{sym}^{2} f\right), \\
& M_{3}:=L\left(\frac{a}{c}, f \times f\right) L\left(\frac{b}{c}, f \times f\right) L\left(1, \operatorname{sym}^{2} f\right),
\end{aligned}
$$

respectively.

We move the integral line of the integral in (28) to the parallel segment with $\mathfrak{R} s=(1 / 2 a)$. We first consider the case $c \leq 2 a$. In this situation, the points $s=(1 / a), s=(1 / b)$, and $s=(1 / c)$ are all poles of the integrand in the rectangle $I_{T}$. Therefore, by Cauchy's residue theorem, we can obtain

$$
\begin{aligned}
S_{f \times f}(a, b, c ; x) \\
=\left\{\operatorname{Res}_{s=(1 / a)}+\operatorname{Res}_{s=(1 / b)}+\operatorname{Res}_{s=(1 / c)}\right\} L(a s, f \times f) L(b s, f \times f) L(c s, f \times f) \frac{x^{s}}{s} \\
\quad+\frac{1}{2 \pi i}\left\{\int_{(1 / 2 a)-i T}^{(1 / 2 a)+i T}+\int_{(1 / 2 a)+i T}^{\eta+i T}+\int_{\eta-i T}^{(1 / 2 a)-i T}\right\} L(a s, f \times f) L(b s, f \times f) L(c s, f \times f) \frac{x^{s}}{s} d s+O\left(\frac{x^{(1 / a)+\varepsilon}}{T}\right) \\
\quad:=M_{1} x^{(1 / a)}+M_{2} x^{(1 / b)}+M_{3} x^{(1 / c)}+J_{1}+J_{2}+J_{3}+O\left(\frac{x^{(1 / a)+\varepsilon}}{T}\right) .
\end{aligned}
$$

Now, the remaining work is to handle these three terms $J_{1}, J_{2}$, and $J_{3}$. In addition, the estimates of these integrals on the horizontal parts are analogous, and so we deal with $J_{2}$ and $J_{3}$ firstly. To estimate $J_{2}$ and $J_{3}$, similarly to the method estimating $G_{2}$ and $G_{3}$, we still divide the integral interval into the following four short intervals $I_{1}^{*}, \ldots, I_{4}^{*}$ and apply Lemmas 3 and 4.

Interval $I_{1}^{*}$ :

$$
\begin{array}{r}
I_{1}^{*}:=\left\{s=\sigma+i T: \frac{1}{2} \leq a \sigma \leq 1, \frac{b}{2 a} \leq b \sigma \leq 1, \frac{c}{2 a} \leq c \sigma \leq 1\right\} \\
=\left\{s=\sigma+i T: \frac{1}{2 a} \leq \sigma \leq \frac{1}{c}\right\} .
\end{array}
$$

In this interval, we have

$$
\begin{aligned}
T^{-1} & \times \int_{I_{1}^{*}} x^{\sigma} \mid \zeta(a \sigma+i a t)\left(L\left(a \sigma+i a t, \operatorname{sym}^{2} f\right) \zeta(b \sigma+i b t) L\left(b \sigma+i b t, \operatorname{sym}^{2} f\right)|\cdot| \zeta(c \sigma+i c t) L\left(c \sigma+i c t, \operatorname{sym}^{2} f\right) \mid \mathrm{d} \sigma\right. \\
& \ll \max _{(1 / 2 a) \leq \sigma \leq(1 / c)} x^{\sigma} T^{((13 / 42)+(5 / 4))(1-a \sigma)} T^{((13 / 42)+(5 / 4))(1-b \sigma)} T^{((13 / 42)+(5 / 4))(1-c \sigma)} T^{-1+\varepsilon} \\
& \ll \max _{(1 / 2 a) \leq \sigma \leq(1 / c)} T^{(309 / 84)+\varepsilon}\left(\frac{x}{T^{(131 / 84)(a+b+c)}}\right)^{\sigma} \\
& \ll x^{1 / c} T^{(89 / 42)-(131(a+b) / 84 c)+\varepsilon}+x^{1 / 2 a} T^{(487 / 168)-(131(b+c) / 168 a)+\varepsilon} .
\end{aligned}
$$

Interval $I_{2}^{*}$ :

$$
\begin{aligned}
I_{2}^{*} & :=\left\{s=\sigma+i T: \frac{1}{2} \leq a \sigma \leq 1, \frac{b}{2 a} \leq b \sigma \leq 1,1<c \sigma \leq c \eta\right\} \\
& =\left\{s=\sigma+i T: \frac{1}{c}<\sigma \leq \frac{1}{b}\right\} .
\end{aligned}
$$


In this interval, we have

$$
\begin{aligned}
T^{-1} & \times \int_{I_{2}^{*}} x^{\sigma} \mid \zeta(a \sigma+i a t)\left(L\left(a \sigma+i a t, \operatorname{sym}^{2} f\right) \zeta(b \sigma+i b t) L\left(b \sigma+i b t, \operatorname{sym}^{2} f\right)|\cdot| \zeta(c \sigma+i c t) L\left(c \sigma+i c t, \operatorname{sym}^{2} f\right) \mid \mathrm{d} \sigma\right. \\
& \ll \max _{(1 / c)<\sigma \leq(1 / b)} x^{\sigma} T^{((13 / 42)+(5 / 4))(1-a \sigma)} T^{((13 / 42)+(5 / 4))(1-b \sigma)} T^{-1+\varepsilon} \\
& \ll \max _{(1 / c)<\sigma \leq(1 / b)} T^{(89 / 42)+\varepsilon}\left(\frac{x}{T^{(131 / 84)(a+b)}}\right)^{\sigma} \\
& \ll x^{1 / b} T^{(47 / 84)-(131 a / 84 b)+\varepsilon}+x^{1 / c} T^{(89 / 42)-(131(a+b) / 84 c)+\varepsilon} .
\end{aligned}
$$

Interval $I_{3}^{*}$ :

$$
\begin{aligned}
I_{3}^{*} & :=\left\{s=\sigma+i T: \frac{1}{2} \leq a \sigma \leq 1,1<b \sigma \leq b \eta, \frac{c}{2 a} \leq c \sigma \leq 1\right\} \\
& =\left\{s=\sigma+i T: \frac{1}{b}<\sigma \leq \frac{1}{c}\right\} .
\end{aligned}
$$

This interval is an empty set noting that $(1 / b)>(1 / c)$.
Interval $I_{4}^{*}$ :

$$
\begin{aligned}
I_{4}^{*} & :=\left\{s=\sigma+i T: \frac{1}{2} \leq a \sigma \leq 1,1<b \sigma \leq b \eta, 1<c \sigma \leq c \eta\right\} \\
& =\left\{s=\sigma+i T: \frac{1}{b}<\sigma \leq \frac{1}{a}\right\} .
\end{aligned}
$$

In this interval, we have

$$
\begin{aligned}
T^{-1} & \times \int_{I_{4}^{*}} x^{\sigma} \mid \zeta(a \sigma+i a t)\left(L\left(a \sigma+i a t, \operatorname{sym}^{2} f\right) \zeta(b \sigma+i b t) L\left(b \sigma+i b t, \operatorname{sym}^{2} f\right)|\cdot| \zeta(c \sigma+i c t) L\left(c \sigma+i c t, \operatorname{sym}^{2} f\right) \mid \mathrm{d} \sigma\right. \\
& \ll \max _{(1 / b)<\sigma \leq(1 / a)} x^{\sigma} T^{((13 / 42)+(5 / 4))(1-a \sigma)} T^{-1+\varepsilon} \\
& \ll x^{1 / a} T^{-1+\varepsilon}+x^{1 / b} T^{(47 / 84)-(131 a / 84 b)+\varepsilon} .
\end{aligned}
$$

From (55)-(60), we can obtain

$$
\begin{aligned}
\mid J_{2} & +J_{3} \mid \\
& \ll T^{-1} \int_{1 / 2 a}^{\eta} x^{\sigma} \mid \zeta(a \sigma+i a t)\left(L\left(a \sigma+i a t, \operatorname{sym}^{2} f\right) \zeta(b \sigma+i b t) L\left(b \sigma+i b t, \operatorname{sym}^{2} f\right)|\cdot| \zeta(c \sigma+i c t) L\left(c \sigma+i c t, \operatorname{sym}^{2} f\right) \mid \mathrm{d}\right. \\
& =T^{-1} \int_{I_{1}^{*} \cup \cdots \cup I_{4}^{*}} x^{\sigma} \mid \zeta(a \sigma+i a t)\left(L\left(a \sigma+i a t, \operatorname{sym}^{2} f\right) \zeta(b \sigma+i b t) L\left(b \sigma+i b t, \operatorname{sym}^{2} f\right)|\cdot| \zeta(c \sigma+i c t) L\left(c \sigma+i c t, \operatorname{sym}^{2} f\right) \mid \mathrm{d} \sigma\right. \\
& \ll x^{1 / 2 a} T^{(487 / 168)-(131(b+c) / 168 a)+\varepsilon}+x^{1 / b} T^{(47 / 84)-(131 a / 84 b)+\varepsilon}+x^{1 / c} T^{(89 / 42)-(131(a+b) / 84 c)+\varepsilon}+x^{(1 / a)+\varepsilon} T^{-1+\varepsilon} .
\end{aligned}
$$

Now, we turn to estimate $J_{1}$, and we have

$$
\begin{aligned}
\left|J_{1}\right| & \ll x^{1 / 2 a} \int_{1}^{T}\left|L\left(\frac{1}{2}+i a t, f \times f\right) L\left(\frac{b}{2 a}+i b t, f \times f\right) L\left(\frac{c}{2 a}+i c t, f \times f\right)\right| t^{-1} \mathrm{~d} t+x^{1 / 2 a} \\
& \ll x^{1 / 2 a}+x^{1 / 2 a} \log T \max _{T_{1} \leq T} T_{1}^{-1} \int_{T_{1} / 2}^{T_{1}}\left|\zeta\left(\frac{1}{2}+i a t\right) L\left(\frac{1}{2}+i a t, \operatorname{sym}^{2} f\right)\right| \\
& \cdot\left|\zeta\left(\frac{b}{2 a}+i b t\right) L\left(\frac{b}{2 a}+i b t, \operatorname{sym}^{2} f\right) \zeta\left(\frac{c}{2 a}+i c t\right) L\left(\frac{c}{2 a}+i c t, \operatorname{sym}^{2} f\right)\right| \mathrm{d} t .
\end{aligned}
$$


Then, using Lemmas 3 and 4 and Hölder's inequality, we obtain

$$
\begin{aligned}
\left|J_{1}\right| \ll & x^{1 / 2 a}+x^{1 / 2 a} \log T \max _{T_{1} \leq T} T_{1}^{-1} T_{1}^{(13 / 42)(1-(b / 2 a))} T_{1}^{(5 / 4)(1-(b / 2 a))} T_{1}^{(13 / 42)(1-(c / 2 a))} T_{1}^{(5 / 4)(1-(c / 2 a))} \\
& \times \int_{T_{1} / 2}^{T_{1}}\left|\zeta\left(\frac{1}{2}+i a t\right) L\left(\frac{1}{2}+i a t, \operatorname{sym}^{2} f\right)\right| \mathrm{d} t \\
& \ll x^{1 / 2 a}+x^{1 / 2 a} \log T \max _{T_{1} \leq T} T_{1}^{-1+\varepsilon} T_{1}^{(13 / 42)(1-(b / 2 a))} T_{1}^{(5 / 4)(1-(b / 2 a))} T_{1}^{(13 / 42)(1-(c / 2 a))} T_{1}^{(5 / 4)(1-(c / 2 a))} \\
& \times\left(\int_{T_{1} / 2}^{T_{1}}\left|\zeta\left(\frac{1}{2}+i a t\right)\right|^{12} \mathrm{~d} t\right)^{1 / 12}\left(\int_{T_{1} / 2}^{T_{1}}\left|L\left(\frac{1}{2}+i a t, \operatorname{sym}^{2} f\right)\right|^{2} \mathrm{~d} t\right)^{1 / 2}\left(\int_{T_{1} / 2}^{T_{1}} 1 \mathrm{~d} t\right)^{5 / 12} \\
& \ll x^{(1 / 2 a)+\varepsilon} T^{(145 / 42)-(131(b+c) / 168 a)+\varepsilon} .
\end{aligned}
$$

By putting (53), (61), and (63) together, we have

$$
\begin{aligned}
& S_{f \times f}(a, b, c ; x) \\
& =M_{1} x^{1 / a}+M_{2} x^{1 / b}+M_{3} x^{1 / c}+O\left(x^{\varepsilon}\left(x^{1 / 2 a} T^{(145 / 42)-(131(b+c) / 168 a)}+x^{1 / b} T^{(47 / 84)-(131 a / 84 b)}+x^{1 / c} T^{(89 / 42)-(131(a+b) / 84 c)}+x^{1 / a} T^{-1+\varepsilon}\right)\right) .
\end{aligned}
$$

By taking $T=x^{(84 / 748 a-131(b+c))}$ in (64), we can obtain

$$
\begin{aligned}
S_{f \times f}(a, b, c ; x)= & M_{1} x^{1 / a}+M_{2} x^{1 / b}+M_{3} x^{1 / c} \\
& +O\left(x^{(1 / a)-(84 / 748 a-131(b+c))+\varepsilon}\right),
\end{aligned}
$$

which proves the first result in Theorem 2 .

For the case $b<2 a<c$, we use a similar argument to the first corresponding case. In this case, the points $s=(1 / a)$ and $s=(1 / b)$ are the two simple poles of the integrand in the rectangle $I_{T}$. Then, by Cauchy's residue theorem we have

$$
\begin{aligned}
& S_{f \times f}(a, b, c ; x) \\
&=\left\{\operatorname{Res}_{s=(1 / a)}+\operatorname{Res}_{s=(1 / b)}\right\} L(a s, f \times f) L(b s, f \times f) L(b s, f \times f) \frac{x^{s}}{s} \\
&+\frac{1}{2 \pi i}\left\{\int_{(1 / 2 a)-i T}^{(1 / 2 a)+i T}+\int_{(1 / 2 a)+i T}^{\eta+i T}+\int_{\eta-i T}^{(1 / 2 a)-i T}\right\} L(a s, f \times f) L(b s, f \times f) L(c s, f \times f) \frac{x^{s}}{s} d s+O\left(\frac{x^{(1 / a)+\varepsilon}}{T}\right) \\
&= M_{1} x^{(1 / a)}+M_{2} x^{(1 / b)}+J_{1}^{\prime \prime}+J_{2}^{\prime \prime}+J_{3}^{\prime \prime}+O\left(\frac{x^{(1 / a)+\varepsilon}}{T}\right) .
\end{aligned}
$$

To estimate $J_{2}^{\prime \prime}+J_{3}^{\prime \prime}$, we still divide the integral interval into four short intervals $I_{1}^{* *}, \ldots, I_{4}^{* *}$, which are different from ones for the case $c \leq 2 a$. In fact, the corresponding short intervals $I_{1}^{* *}, I_{3}^{* *}$ become empty sets in this situation. However, we still can estimate $J_{2}^{\prime \prime}+J_{3}^{\prime \prime}$ by following a similar argument to the corresponding parts of the case $c \leq 2 a$ and get

$$
\begin{aligned}
\left|J_{2}^{\prime \prime}+J_{3}^{\prime \prime}\right| \ll & x^{1 / 2 a} T^{(75 / 56)-(131 b / 168 a)+\varepsilon}+x^{1 / b} T^{(47 / 84)-(131 a / 84 b)+\varepsilon} \\
& +x^{(1 / a)+\varepsilon} T^{-1+\varepsilon} .
\end{aligned}
$$

The estimate of $J_{1}^{\prime \prime}$ becomes the following at the current case by noting $(c / 2 a)>1>(b / 2 a)$. 


$$
\begin{aligned}
\left|J_{1}^{\prime}\right| & \ll x^{1 / 2 a} \int_{1}^{T}\left|\zeta\left(\frac{1}{2}+i a t\right) L\left(\frac{1}{2}+i a t, \operatorname{sym}^{2} f\right) \zeta\left(\frac{b}{2 a}+i b t\right) L\left(\frac{b}{2 a}+i b t, \operatorname{sym}^{2} f\right)\right| \cdot\left|\zeta\left(\frac{c}{2 a}+i c t\right) L\left(\frac{c}{2 a}+i c t, \operatorname{sym}^{2} f\right)\right| t^{-1} \mathrm{~d} t+x^{1 / 2 a} \\
& \ll x^{1 / 2 a} \log T \max _{T_{1} \leq T} T_{1}^{-1+\varepsilon} T_{1}^{(131 / 84)(1-(b / 2 a))} \int_{T_{1} / 2}^{T_{1}}\left|\zeta\left(\frac{1}{2}+i a t\right) L\left(\frac{1}{2}+i a t, \operatorname{sym}^{2} f\right)\right| \mathrm{d} t+x^{1 / 2 a} \\
& \ll x^{1 / 2 a} \log T \max _{T_{1} \leq T} T_{1}^{-1+\varepsilon} T_{1}^{(131 / 84)(1-(b / 2 a))}\left(\int_{T_{1} / 2}^{T_{1}}\left|\zeta\left(\frac{1}{2}+i a t\right)\right|^{12} \mathrm{~d} t\right)^{1 / 12} \times\left(\int_{T_{1} / 2}^{T_{1}}\left|L\left(\frac{1}{2}+i a t, \operatorname{sym}^{2} f\right)\right|^{2} \mathrm{~d} t\right)^{1 / 2}\left(\int_{T_{1} / 2}^{T_{1}} 1 \mathrm{~d} t\right)^{5 / 12}+x^{1 / 2 a} \\
& \ll x^{1 / 2 a} T^{(159 / 84)-(131 b / 168 a)+\varepsilon} .
\end{aligned}
$$

Thus, recalling (67), we have

$$
S_{f \times f}(a, b, c ; x)=M_{1} x^{1 / a}+M_{2} x^{1 / b}+O\left(x^{1 / a-(84 / 486 a-131 b)+\varepsilon}\right),
$$

$$
\begin{aligned}
S_{f \times f}(a, b, c ; x)= & M_{1} x^{1 / a}+M_{2} x^{1 / b} \\
& +O\left(x^{1 / 2 a} T^{(159 / 84)-(131 b / 168 a)+\varepsilon}\right. \\
& \left.+x^{1 / b} T^{(47 / 84)-(131 a / 84 b)+\varepsilon}+x^{(1 / a)+\varepsilon} T^{-1+\varepsilon}\right) .
\end{aligned}
$$

which proves the second result of Theorem 2 .

For the case $2 a \leq b$, we use a similar argument to the first corresponding case. In this situation, the point $s=(1 / a)$ is the only simple pole of the integrand in the rectangle $I_{T}$. Then, Cauchy's residue theorem shows

By taking $T=x^{(84 / 486 a-131 b)}$ in (69), we have

$$
\begin{aligned}
S_{f \times f}(a, b, c ; x) \\
=\operatorname{Res}_{s=(1 / a)} L(a s, f \times f) L(b s, f \times f) L(c s, f \times f) \frac{x^{s}}{s}+O\left(\frac{x^{(1 / a)+\varepsilon}}{T}\right) \\
\quad+\frac{1}{2 \pi i}\left\{\int_{(1 / 2 a)-i T}^{(1 / 2 a)+i T}+\int_{(1 / 2 a)+i T}^{\eta+i T}+\int_{\eta-i T}^{(1 / 2 a)-i T}\right\} L(a s, f \times f) L(b s, f \times f) L(c s, f \times f) \frac{x^{s}}{s} d s \\
=M_{1} x^{(1 / a)}+J_{1}^{\prime}+J_{2}^{\prime}+J_{3}^{\prime}+O\left(\frac{x^{(1 / a)+\varepsilon}}{T}\right) .
\end{aligned}
$$

To estimate $J_{2}^{\prime}+J_{3}^{\prime}$, we still divide the integral interval into four short intervals $I_{1}^{* * *}, \ldots, I_{4}^{* * *}$, which are different from ones for the case $c \leq 2 a$. In fact, the corresponding short intervals $I_{1}^{* * *}, I_{2}^{* * *}, I_{3}^{* * *}$ become empty sets in this situation. However, we still can estimate $J_{2}^{\prime}+J_{3}^{\prime}$ by following a similar argument to the corresponding parts of the case $c \leq 2 a$ and get

$$
\left|J_{2}^{\prime}+J_{3}^{\prime}\right| \ll x^{1 / 2 a} T^{-(37 / 168)+\varepsilon}+x^{(1 / a)+\varepsilon} T^{-1+\varepsilon}
$$


The estimate of $J_{1}^{\prime}$ becomes the following by noting $(c / 2 a)>(b / 2 a)>1$.

$$
\begin{aligned}
\left|J_{1}^{\prime}\right| \ll & x^{1 / 2 a} \int_{1}^{T}\left|\zeta\left(\frac{1}{2}+i a t\right) L\left(\frac{1}{2}+i a t, \operatorname{sym}^{2} f\right) \zeta\left(\frac{b}{2 a}+i b t\right) L\left(\frac{b}{2 a}+i b t, \operatorname{sym}^{2} f\right)\right| \cdot\left|\zeta\left(\frac{c}{2 a}+i c t\right) L\left(\frac{c}{2 a}+i c t, \mathrm{sym}^{2} f\right)\right| t^{-1} \mathrm{~d} t+x^{1 / 2 a} \\
& \ll x^{1 / 2 a} \log T \max _{T_{1} \leq T} T_{1}^{-1+\varepsilon} \int_{T_{1} / 2}^{T_{1}}\left|\zeta\left(\frac{1}{2}+i a t\right) L\left(\frac{1}{2}+i a t, \operatorname{sym}^{2} f\right)\right| \mathrm{d} t+x^{1 / 2 a} \\
& \ll x^{1 / 2 a} \log T \max _{T_{1} \leq T} T_{1}^{-1+\varepsilon}\left(\int_{T_{1} / 2}^{T_{1}}\left|\zeta\left(\frac{1}{2}+i a t\right)\right|^{12} \mathrm{~d} t\right)^{1 / 12} \\
& \times\left(\int_{T_{1} / 2}^{T_{1}}\left|L\left(\frac{1}{2}+i a t, \operatorname{sym}^{2} f\right)\right|^{2} \mathrm{~d} t\right)^{1 / 2}\left(\int_{T_{1} / 2}^{T_{1}} 1 \mathrm{~d} t\right)^{5 / 12}+x^{1 / 2 a} \\
& \ll x^{1 / 2 a} T^{(1 / 3)+\varepsilon} .
\end{aligned}
$$

Thus, recalling (71), we have

$$
S_{f \times f}(a, b, c ; x)=M_{1} x^{1 / a}+O\left(x^{1 / 2 a} T^{(1 / 3)+\varepsilon}+x^{(1 / a)+\varepsilon} T^{-1}\right) .
$$

By taking $T=x^{(3 / 8 a)}$ in (74), we have

$$
S_{f \times f}(a, b, c ; x)=M_{1} x^{1 / a}+O\left(x^{(5 / 8 a)+\varepsilon}\right),
$$

which proves the third result of Theorem 2 .

\section{Data Availability}

The data supporting the findings of this study are included within the article.

\section{Conflicts of Interest}

The authors declare that they have no conflicts of interest.

\section{Acknowledgments}

This work was supported by National Natural Science Foundation of China (Grant nos. 11801328 and 11771256).

\section{References}

[1] P. Deligne, "La conjecture de Weil," Institut des Hautes Études Scientifiques Publications Mathematiques, vol. 43, pp. 29-39, 1974.

[2] H. Iwaniec, "Topics in classical automorphic forms," in Graduate Studies in MathematicsVol. 17, American Mathematical Society, Providence, RI, USA, 1997.

[3] S. Gelbart and H. Jacquet, "A relation between automorphic representations of GL(2) and GL(3)," Annales Scientifiques De l'École Normale Supérieure, vol. 11, no. 4, pp. 471-542, 1978.
[4] H. Kim, "Functoriality for the exterior square of $G L_{4}$ and symmetric fourth of $G L_{2}$," Journal of the American Mathematical Society, vol. 16, pp. 139-183, 2003.

[5] H. H. Kim and F. Shahidi, "Functorial products for $G L 2 \times G L$ 3 and the symmetric cube for GL 2," The Annals of Mathematics, vol. 155, no. 3, pp. 837-893, 2002.

[6] H. H. Kim and F. Shahidi, "Cuspidality of symmetric powers with applications," Duke Mathematical Journal, vol. 112, pp. 177-197, 2002.

[7] A. Perelli, "General L-functions," Annali di Matematica Pura ed Applicata, vol. 130, no. 1, pp. 287-306, 1982.

[8] L. Barthel and D. Ramakrishnan, "A nonvanishing result for twists of $L$-functions of GL(n)," Duke Mathematical Journal, vol. 74, pp. 681-700, 1994.

[9] K. Chandrasekharan and R. Narasimhan, "Functional equations with multiple gamma factors and the average order of arithmetical functions," The Annals of Mathematics, vol. 76, no. 1, pp. 93-136, 1962.

[10] L. Dong, H. Liu, and D. Zhang, "Zero density estimates for automorphic $L$-functions of $S L(2, Z)$," Acta Mathematica Hungarica, vol. 148, no. 1, pp. 191-210, 2016.

[11] A. Good, "The square mean of Dirichlet series associated with cusp forms," Mathematika, vol. 29, no. 2, pp. 278-295, 1982.

[12] J. L. Hafner and A. Ivić, “"On sums of Fourier coefficients of cusp forms," L'Enseignement Mathématique, vol. 35, pp. 375-382, 1989.

[13] Y. Jiang, G. Lü, and X. Yan, "Mean value theorem connected with Fourier coefficients of Hecke-Maass forms for $S L$ $(m, \mathbb{Z})$," Mathematical Proceedings of the Cambridge Philosophical Society, vol. 161, no. 2, pp. 339-356, 2016.

[14] H. Lao, "On the fourth moment of coefficients of symmetric square L-function," Chinese Annals of Mathematics, Series B, vol. 33, no. 6, pp. 877-888, 2012.

[15] H. X. Lao, "On comparing Hecke eigenvalues of cusp forms," Acta Mathematica Hungarica, vol. 160, no. 1, pp. 58-71, 2020.

[16] H. X. Lao, "The cancellation of Fourier coefficients of cusp forms over different sparse sequences," Acta Mathematica Sinica, English Series, vol. 29, no. 10, pp. 1963-1972, 2013. 
[17] H. Lao, "Mean value of Dirichlet series coefficients of RankinSelberg L-functions," Lithuanian Mathematical Journal, vol. 57, no. 3, pp. 351-358, 2017.

[18] H. Lao and H. Wei, " $\Omega$-result on coefficients of automorphic $L$-functions over sparse sequences," Journal of the Korean Mathematical Society, vol. 52, no. 5, pp. 945-954, 2015.

[19] R. M. Nunes, "On the subconvexity estimate for self-dual $G L$ (3) $L$-functions in the $t$-aspect," 2017, https://arxiv.org/ pdf/1703.04424.

[20] R. A. Rankin, "Sums of cusp form coefficients," in Automorphic Forms and Analytic Number Theory (Montreal, PQ, 1989), pp. 115-121, University Montreal, Montreal, Canada, 1990.

[21] R. A. Rankin, “Contributions to the theory of Ramanujan's function $\tau(n)$ and similar arithmetical functions," Mathematical Proceedings of the Cambridge Philosophical Society, vol. 35, no. 3, pp. 357-372, 1939.

[22] A. Selberg, "Bemerkungen ber eine dirichletsche reihe, die mit der theorie der modulformen nahe verbunden ist," Archiv der Mathematik, vol. 43, pp. 47-50, 1940.

[23] P. Song, W. Zhai, and D. Zhang, "Power moments of Hecke eigenvalues for congruence group," Journal of Number Theory, vol. 198, pp. 139-158, 2019.

[24] J. Wu, "Power sums of Hecke eigenvalues and application," Acta Arithmetica, vol. 137, no. 4, pp. 333-344, 2009.

[25] Y. Ye and D. Zhang, "Zero density for automorphic $L$ functions," Journal of Number Theory, vol. 133, no. 11, pp. 3877-3901, 2013.

[26] D. Zhang, Y.-K. Lau, and Y. Wang, "Remark on the paper "on products of Fourier coefficients of cusp forms"' Archiv der Mathematik, vol. 108, no. 3, pp. 263-269, 2017.

[27] D. Zhang and Y. Wang, "Ternary quadratic form with prime variables attached to Fourier coefficients of primitive holomorphic cusp form," Journal of Number Theory, vol. 176, pp. 211-225, 2017.

[28] D. Zhang and Y. Wang, "Higher-power moments of Fourier coefficients of holomorphic cusp forms for the congruence subgroup $\Gamma_{0}(N)$," The Ramanujan Journal, vol. 47, no. 3, pp. 685-700, 2018.

[29] R. Zhang, X. Han, and D. Y. Zhang, "Power moments of the Riesz mean error term of symmetric square $L$-function in short intervals," Symmetry, vol. 12, 2020.

[30] E. Hecke, "Theorie der Eisensteinschen Reihen höherer Stufe und ihre Anwendung auf Funktionentheorie und Arithmetik," Abhandlungen aus dem Mathematischen Seminar der Universität Hamburg, vol. 5, no. 1, pp. 199-224, 1927.

[31] S. Kanemitsu, A. Sankaranarayanan, and Y. Tanigawa, "A mean value theorem for Dirichlet series and a general divisor problem," Monatshefte Für Mathematik, vol. 136, no. 1, pp. 17-34, 2002.

[32] O. M. Fomenko, "Mean value theorems for a class of Dirichlet series," Journal of Mathematical Sciences (New York), vol. 157, pp. 659-672, 1999.

[33] G. Lü, "On general divisor problems involving Hecke eigenvalues," Acta Mathematica Hungarica, vol. 135, no. 1-2, pp. 148-159, 2012.

[34] J. Huang, H. F. Liu, and F. X. Xu, "“Two-dimension divisor problems related to symmetric $L$-function," Symmetry, vol. 13, 2021.

[35] H. F. Liu, "Mean value estimates of the coefficients of product L-functions," Acta Mathematica Hungarica, vol. 156, no. 1, pp. 102-111, 2018.
[36] H. F. Liu and R. Zhang, "Some problems involving Hecke eigenvalues," Acta Mathematica Hungarica, vol. 159, no. 1, pp. 287-298, 2019.

[37] G. S. Lü and A. Sankaranarayanan, "On the coefficients of triple product L-functions," Rocky Mountain Journal of Mathematics, vol. 47, pp. 553-570, 2017.

[38] A. Ivić, The Riemann Zeta-Function, Theory and Applications, Dover Publicatios, Inc., Mineola, NY, USA, 2003.

[39] D. R. Heath-Brown, "The twelfth power moment of the Riemann-function," The Quarterly Journal of Mathematics, vol. 29, no. 4, pp. 443-462, 1978.

[40] J. Bourgain, "Decoupling, exponential sums and the Riemann zeta function," American Mathematical Society, vol. 30, pp. 225-224, 2017.

[41] H. Iwaniec and E. Kowalski, Analytic Number Theory. American Mathematical Society Colloquium PublicationsVol. 53, American Mathematical Society, Providence, RI, USA, 2004. 